\title{
GMR
}

\section{Molecular characterization, tissue expression profile, and SNP analysis of porcine SLC13A5}

\author{
L.Y. Wang, J. Jiang and H.M. Ma
}

College of Animal Science and Technology of Hunan Agricultural University, Changsha, China

Corresponding authors: J. Jiang / H.M. Ma

E-mail: jiangjun1121@126.com / mahaiming2000@163.com

Genet. Mol. Res. 14 (4): 16090-16101 (2015)

Received August 22, 2015

Accepted October 2, 2015

Published December 7, 2015

DOI http://dx.doi.org/10.4238/2015.December.7.21

\begin{abstract}
Solute carrier family 13 (sodium-dependent citrate transporter member 5, SLC13A5) gene has been recently found to play an important role in intramuscular fat content in pigs. In this study, the full-length cDNA of porcine SLC13A5 was obtained from the longissimus dorsi muscle of Shaziling pigs, using the rapid amplification of cDNA ends technique. Full-length porcine SLC13A5 cDNA was $2118 \mathrm{bp}$, with a 1665-bp open reading frame encoding 554 amino acids. The porcine SLC13A5 protein was analyzed using bioinformatic methodology, and found to include 18 potential phosphorylation sites (including six serine, nine threonine, and three tyrosine) and eight putative transmembrane domains. One single nucleotide polymorphism (SNP) site, A251G, was identified by polymerase chain reaction (PCR)-restriction fragment
\end{abstract}


length polymorphism and the associations of this SNP with age at 100 $\mathrm{kg}$ and corrected back fat thickness were found to be not significant. Expression of SLC13A5 was evaluated in ten tissues from 25-day-old full-sib Yorkshire and Shaziling piglets (both $N=3$ ), using quantitative PCR analysis. Expression levels of SLC13A5 differed significantly between the breeds in cecum, liver and crureus muscle. In each breed, gene expression levels were significantly different in longissimus dorsi muscle, compared to the nine other tissues. This study has laid the foundation for further investigations of the molecular mechanisms of SLC13A5 in pigs.

Key words: Pig; SLC13A5; Cloning; Single nucleotide polymorphism; Expression profiles

\section{INTRODUCTION}

It has been previously demonstrated that the solute carrier family 13 member 5 (SLC13A5) gene family consists of five related members including SLC13A1, SLC13A2, SLC13A3, SLC13A4, and SLC13A5. It have been described in a variety of animals, plants, yeast, and bacteria (Shulman and Helfand, 2011; Birkenfeld et al., 2011; Martinez-Beamonte et al., 2011). SLC13A5 is a sodium- coupled transporter that mediates cellular uptake of citrate, which plays important role in the synthesis of fatty acids (Li et al., 2015). Mammalian solute carrier family 13 mediates transport of $\mathrm{Na}^{+}$-coupled anion at the plasma membrane of epithelial (kidney, small intestine, placenta, and liver) (Pajor, 2006; Bergeron et al., 2013). CpG sites of SLC13A5 in glioblastomas exhibited that expression level had a negative correlation with promoter methylation (Etcheverry et al., 2010).

In recent years, more and more researches have been focused on the meat quality. It is a very complicated trait because this trait is made up with many components including the intramuscular fat content (IMF) (Chen et al., 2013; Ros- Freixedes et al., 2014; Sato et al., 2014; Ren et al., 2014). IMF is one of the most important factors affecting meat juiciness, tenderness, and flavor (Schwab et al., 2006; Wood et al., 2004, 2008; Madeira et al., 2013). Estimated heritability of the IMF was 0.78 (Biermann et al., 2015). Schworer and Lorenz (1995) reported that the selection of high IMF in pigs is necessary to improve pork quality. In pigs the H-FABP gene contributed to IMF and it has been applied to breeding programs of pigs (Ding et al., 2011).

The porcine SLC13A5 gene is located on Sus scrofa chromosome 12 (SSC12) (http:// www.ncbi.nlm.nih.gov/projects/mapview/map_search.cgi?taxid=9823\&build=2.1). There are three quantitative trait loci for IMF on SSC12 (Xiong et al., 2012; Ma et al., 2009, 2013). Morever, Luo et al. (2012) used a genome-wide association study (GWAS) method to determine that porcine SLC13A5 was significantly related to IMF.

In the current study, in order to investigate whether SLC13A5 expression was associated with IMF in pigs, the cDNA sequence of SLC13A5 was cloned by rapid amplification of cDNA ends (RACE). The characteristics of the SLC13A5 protein were evaluated using bioinformatic analysis, and expression levels in ten tissues from two pig breeds were determined by quantitative realtime PCR. The aim of this research was to reveal the influence of SLC13A5 on fat deposition and provide further insights into the mechanism of porcine $S L C 13 A 5$ in meat quality trait. 


\section{MATERIAL AND METHODS}

\section{Animals and samples}

Animals were reared in Taoyuan, Ningxiang, Xiangtan, and the Changsha county of Hunan Province, China. Ear tissue samples were collected from adult Ningxiang $(\mathrm{N}=65)$, Yorkshire $(N=330)$, Taoyuan black $(N=112)$, Shaziling $(N=57)$, and Daweizi $(N=80)$ pigs and genomic DNA extractions was performed. Samples of longissimus dorsi, crureus, lung, liver, pancreas, intestine, cecum, heart, spleen, and kidney were collected from 25-day-old full-sib Shazhiling and Yorkshire pigs (both $\mathrm{N}=3$ ), immediately frozen in liquid nitrogen, and then stored at $-80^{\circ} \mathrm{C}$ until further use.

\section{RNA extraction, reverse transcription, cloning and sequencing}

Total RNA was extracted from longissimus dorsi muscle of Shaziling pigs using an RNA extraction kit (Takara, Shiga, Japan) in accordance with the manufacturer instructions. The quality and concentration of RNA in each sample were evaluated by Nanodrop 2000 spectrophotometer. First-strand cDNA was produced using the Superscript II RT reverse transcription system kit (Invitrogen, Carlsbad, CA, USA) in accordance with the manufacturer protocol. Amplification by PCR was conducted under the following conditions: $94^{\circ} \mathrm{C}$ for $5 \mathrm{~min} ; 32$ cycles of $94^{\circ} \mathrm{C}$ for $20 \mathrm{~s}$, $55^{\circ} \mathrm{C}$ for $25 \mathrm{~s}$, and $72^{\circ} \mathrm{C}$ for $30 \mathrm{~s}$; and $72^{\circ} \mathrm{C}$ for $5 \mathrm{~min}$. Following gel purification, RT-PCR products were cloned into a pMD19-T vector (Takara) and sequenced.

\section{Cloning of porcine SLC13A5}

To obtain full-length porcine SLC13A5 cDNA, two primer pairs were designed to amplify 5'- and 3'-ends of SLC13A5 gene, by RACE, and obtained a coding sequence (CDS) of porcine SLC13A5. Amplifications by 5'- and 3'-RACE PCR were performed using a SMART TM RACE cDNA amplification kit (Clontech Company, Madison, WI, USA) and the manufacturer protocol. Gene-specific (GSPs), universal (UPM) and abridged universal amplification (AUAP) primers are presented in Table 1. Amplified products were separated by electrophoresis on a $1.5 \%$ agarose $(\mathrm{w} / \mathrm{v})$ gel. Following gel purification, RT-PCR products were cloned into a pMD18-T vector (Takara) and sequenced, using the DNAStar 7.1 software (http://www. gpxz.com) to obtain full-length cDNA sequence. Sequence homologies were performed by comparisons with published sequences, using the NCBI-BLAST database (http://blast.ncbi. nlm.nih.gov/Blast.cgi).

\section{Bioinformatic analysis}

Analysis of cDNA and amino acid sequence deduction were performed with the Expert protein analysis system (http://www.expasy.org/). Amino acid sequences were aligned using CLUSTALW version 2.0 (http://www.simgene.com/ClustalW), and protein domain features was predicted using the simple modular architecture tool (http://smart.embl-heidelberg.de/). Phosphorylation sites were analyzed by NetPhos2.0 (http://www.cbs.dtu.dk/services/NetPhos/). A phylogenetic tree was constructed with SLC13A5 full-length cDNA sequences of different species using the neighbor-joining method and the MEGA version 5.0 software (Tamura et al., 2011). 


\section{Real-time quantitative PCR (qPCR) analysis}

To evaluate the expression of porcine SLC13A5 in different tissues, RNA was isolated from ten different tissues using an RNA extraction kit and following the manufacturer instructions (Takara). Total cDNA was generated using a QuantiTect transcription kit (Takara). Primers of SYBR green were designed using the Primer premier 5.0 software (Table 1). Real-time PCR was carried out using a BIO-RAD iCycler Thermal Cycler w/iQ5 (Hercules, CA, USA). Results were calculated using the relative quantification method and glyceraldehyde 3-phosphate dehydrogenase $(G A P D H)$ as an internal control gene. Expression levels of SLC13A5 were calculated using the $2^{-\Delta \Delta \mathrm{Ct}}$ comparative CT method $\left(\Delta \Delta \mathrm{Ct}=\Delta \mathrm{Ct}_{\text {target gene }}-\Delta \mathrm{Ct}_{\text {housekeeping gene }}\right)$. Amplification reactions were carried out in a final volume of $20 \mu \mathrm{L}$, comprising $10 \mu \mathrm{L} 2 \mathrm{X}$ qPCR Mix (Takara); $1 \mu \mathrm{L}$ of each primer $(10 \mu \mathrm{M}) ; 1 \mu \mathrm{L}$ template cDNA; and $8 \mu \mathrm{L} \mathrm{ddH}_{2} \mathrm{O}$, according to the following program: $95^{\circ} \mathrm{C}$ for $2 \mathrm{~min}$, followed by 35 cycles of $95^{\circ} \mathrm{C}$ for $10 \mathrm{~s}, 60^{\circ} \mathrm{C}$ for $40 \mathrm{~s}$ and $72^{\circ} \mathrm{C}$ for $30 \mathrm{~s}$.

\section{Single nucleotide polymorphism (SNP) identification and allele frequency analysis of porcine SLC13A5}

Pig genomic DNA was extracted by the phenol/chloroform method from ear tissue samples, and used as template DNA for amplification using five primer pairs (Table 1) to detect SNPS. Genomic DNA was amplified by PCR, and then PCR products were directly sequenced to detect mutations. A polymorphism site was identified using the Seqman program of DNAStar 7.1 software (http://www.gpxz.com). Genotyping was based on genomic DNA of all breeds using the PCRrestriction fragment length polymorphism technique with Bsu36I restriction enzyme (Fermentas, Beijing, China). Amplified PCR products were digested at $37^{\circ} \mathrm{C}$ for $10 \mathrm{~h}$ in a mixture $(20 \mu \mathrm{L})$ containing $9.8 \mu \mathrm{L}$ PCR products; $8 \mu \mathrm{L}$ nuclease-free water; $2 \mu \mathrm{L}$ 10X Buffer Tango (Promega, Madison, WI, USA); and $0.2 \mu \mathrm{L} \mathrm{Bsu36I} \mathrm{(10} \mathrm{U).}$

\section{RESULTS}

\section{cDNA cloning and sequence analysis of porcine SLC13A5}

The complete cDNA sequence of SLC13A5 from Shaziling pigs was obtained by RACE and was 2118 bp long. Porcine SLC13A5 sequence was deposited in GenBank (accession No. KF728381). The total cDNA length and deduced amino acid sequences are displayed in Figure 1. The nucleotide sequence of SLC13A5 contained an open reading frame that was $1665 \mathrm{bp}$ in length and encoded 554 amino acids, and the 5'- and 3'-untranslated regions were 557 and $1320 \mathrm{bp}$ in size, respectively. The predicted molecular weight of SLC13A5 was approximately $61183.7 \mathrm{kDa}$ and the theoretical isoelectric point was 7.45. Among 20 amino acids encoded by the SLC13A5 protein, Leu was in the highest proportion and accounted for $13.4 \%$ of amino acids; His had the lowest proportion (1.2\%). Analysis of the primary protein structure revealed that SLC13A5 comprised a single peptide, eight putative transmembrane domains, and a low complexity region with 12 amino acids (Figure 2) that included 18 phosphorylation sites (Ser 6, Thr 9, and Tyr 3). 


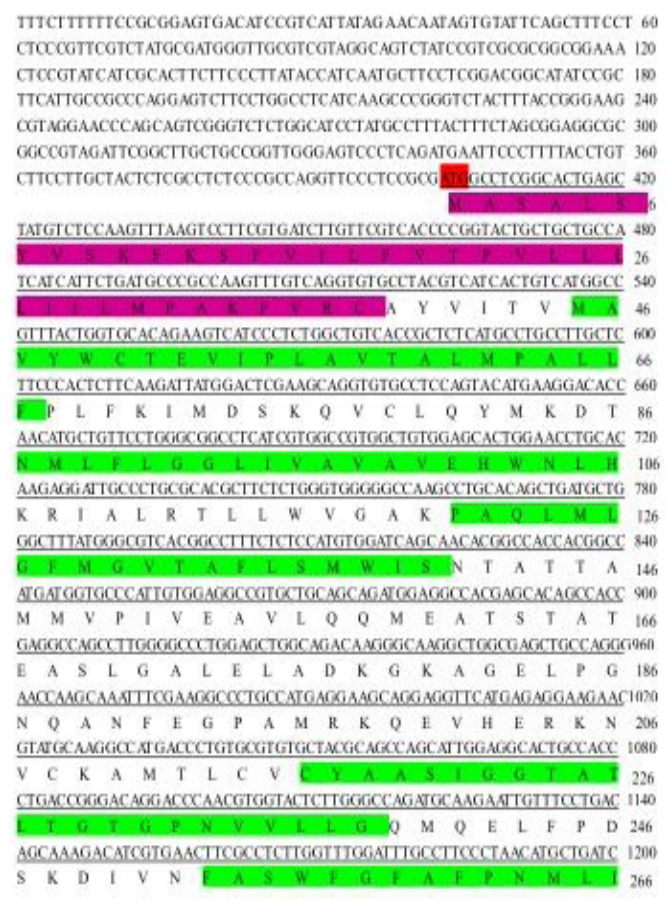

ATGCTGCTOCTCTCCTGGCTGTGGCTACGGTTIATTTATATGIAGIATTCAATATTAAAGAG IZAO

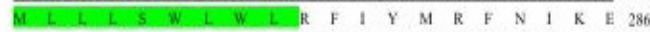

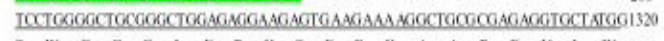

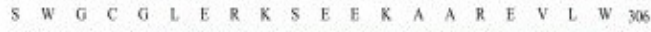

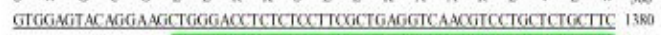
V E Y R K L G P L S F A E V N V L L C I 336

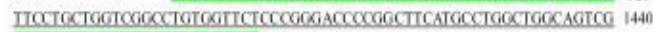
E L L V G L W F S R D P G F M P G W Q S 346 ACACCTGGGTGGAGGGCAAGACAMGITACACCTCTGATGCCACTGTGGCCATCTTCGTG 150 I T W V E G K I S Y Y S D A T V A I I V * GCCTGCTTGCTCTTCATCATGCCTICTGAMAAGGCCTAAGTTCAACTICTCCAGCCAGACT 1560

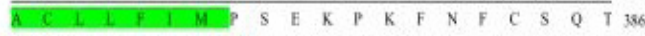

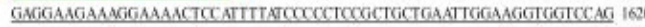

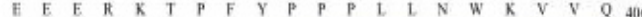
GMGAAAGTACCCTGGGGCATCGTGCTGCTCCTGGGGGGCGGCITTGCTCTGGCTAMAGGA I6SO

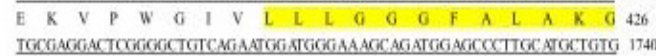
C E GCCCCMGCAGCCATCACCTTGATCTTGTCCTCGCTCATCCCTGTGCTCACTGAGTGCACA 1800

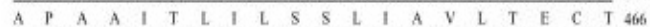
AGCAACGTGGCCACCACTACCCTGTTCCTGCECATCTITGCCTCCATGTCACGTTOCAIT 1860

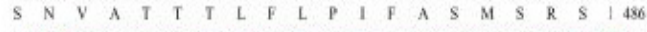
GGOCTCAACCCGCTOTACGTCATGATCOCCTGTACCCTGIAGCTCATCCTITCOCTTCATG 192) 6 L N P L Y Y M I P C T L \& \& \& F A E N 500 CTGCCOGTGGCCACCCCMCCTAATGCCATTGTGTTTTCCTACGGAACACLTCAAGGTTTCC 1980 \begin{tabular}{lllllllllllllllllllll}
\hline L P & Y & A & I & P & P & N & A & I & V & P & S & Y & $G$ & H & L & $K$ & V & $\$$ & 526
\end{tabular} GACATGGTAACTCAGCTGCTITTATTCAATCCTATCAGCTCTCTTOCTTTTCTCATGGIGG 20 HO

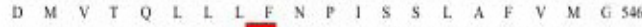

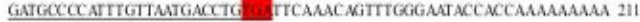
D A P F Y N D L * AMAMAAMAAAMAAAAAAA

Figure 1. cDNA and putative amino acid sequences of porcine SLC13A5 gene and open reading frame of the SLC13A5 nucleotide sequence are underlined; asterisk indicates the stop codon, and start codon and stop codon are shaded with red; the signal peptide is shaded with purple; one low-complexity region is shaded with yellow; eight putative transmembrane domains is shaded with green.

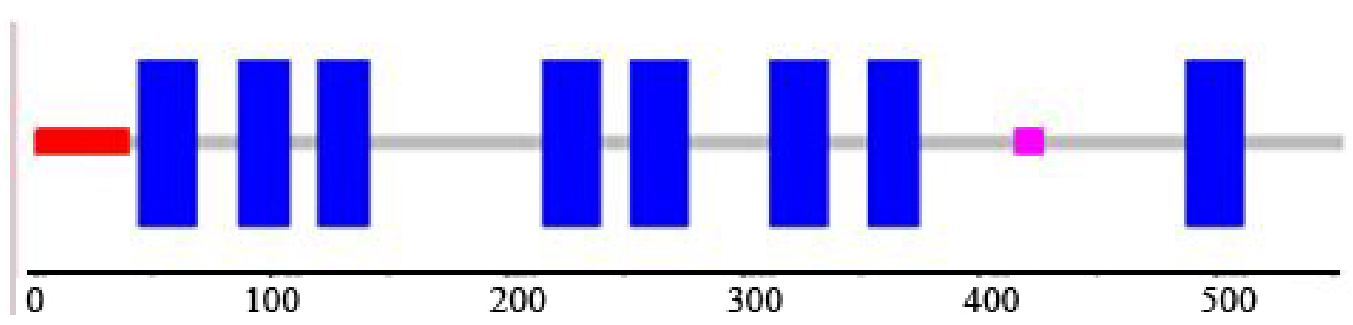

Figure 2. SLC13A5 conservative structure domain analysis in pig.

\section{Deduced protein sequence analysis, putative secondary and tertiary structures, and phylogenetic tree analysis}

A comparison of porcine SLC13A5 with sequences from 13 other animal species was carried out using the DNAMAN software; results are shown in Figure 3. Secondary and tertiary 
protein structure predictions are presented in, Figures 4 and 5 respectively. Based on phylogenetic tree analysis, porcine SLC13A5 sequence clustered with those of the sheep and the cattle, followed by SLC13A5 sequence from the Rhesus, the African clawed frog, the wild pigeon, and the ground tid (Figure 6).

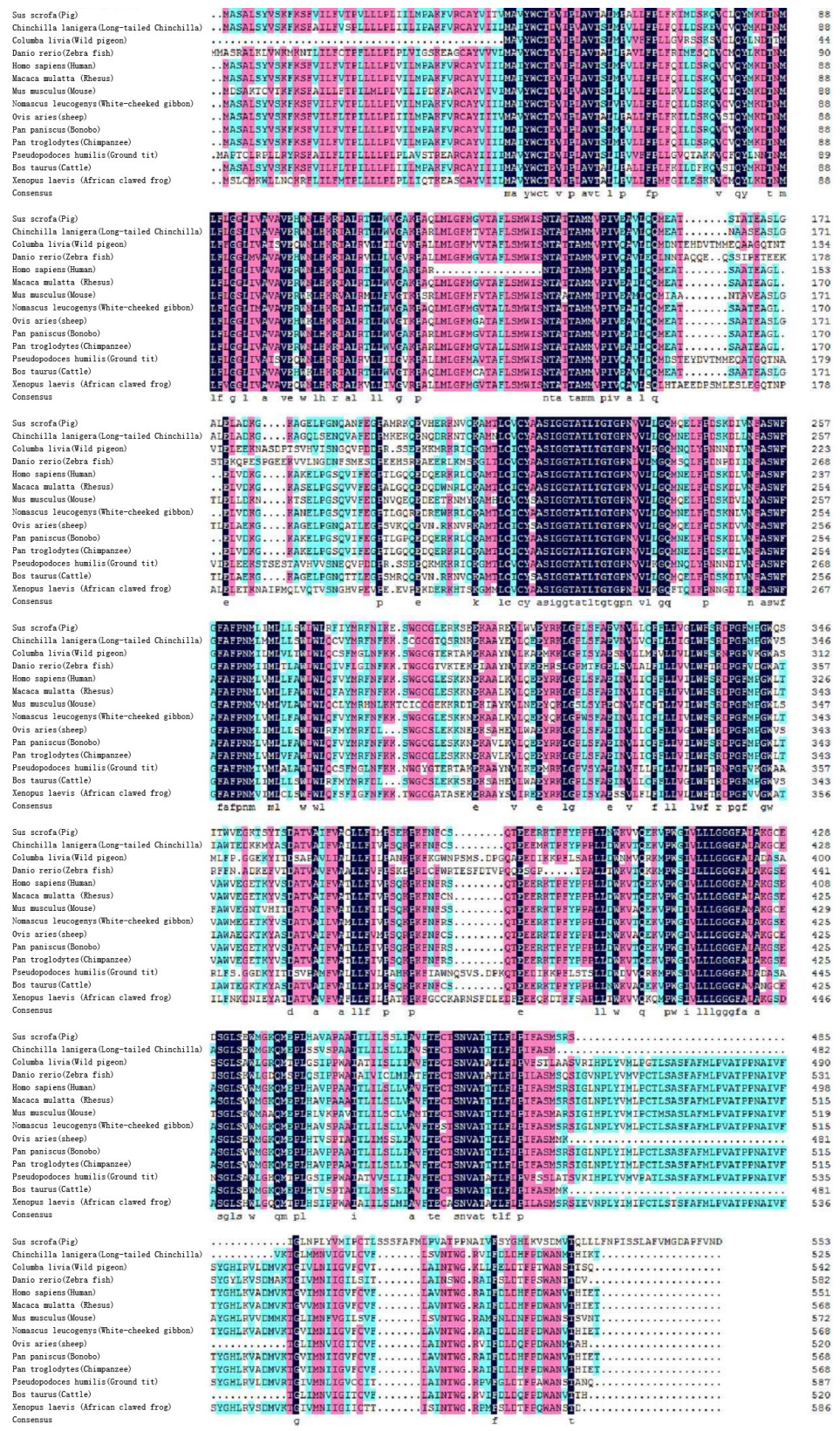

Figure 3. Alignment of amino acid sequences of the SLC1A5 gene in fourteen different breeds. 


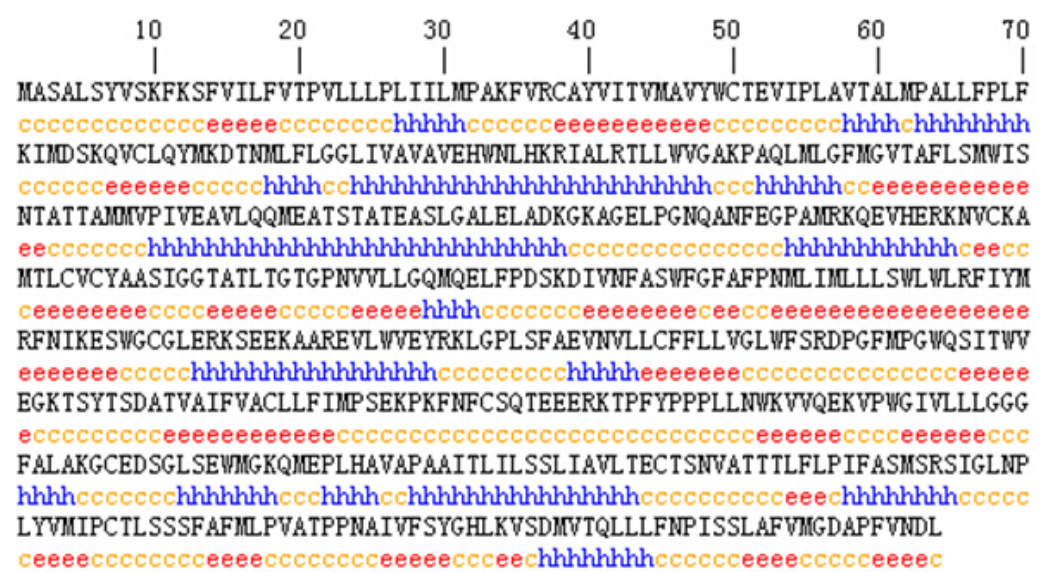

Figure 4. SLC13A5 protein secondary structure prediction based on HNN.

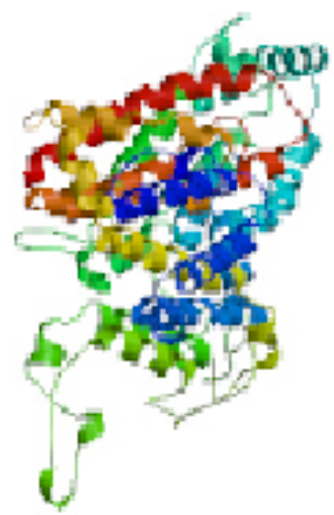

Figure 5. Domain area of $S L C 13 A 5$ protein tertiary structure prediction.

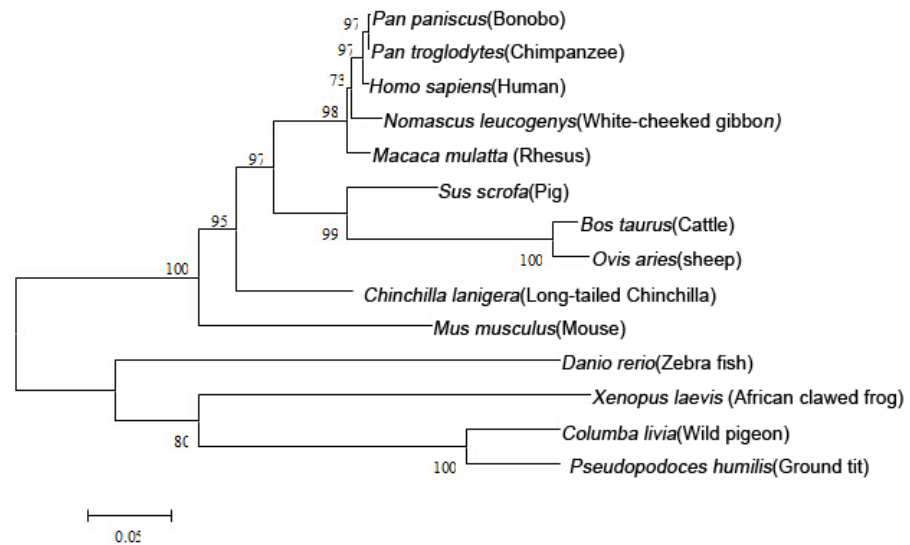

Figure 6. Phylogenetic relationship among the $S L C 13 A 5$ gene of pig and other species. 


\section{Polymorphism detection and allele frequency}

In this study, an SNP site was detected in intron 12 (Table 1). This mutation was reported for Sus scrofa (GenBank accession No. rs319369248) and its gene frequency was not reported. A nucleotide substitution of $A$ by $G$ was observed at the 251 position of the 815-bp fragment amplified with Bsu36I restriction endonuclease (Figure 7). Three genotypes, $A A, A G$ and $G G$, were generated (Figure 8). Allele $G$ was predominant in Ningxiang and Shaziling pigs, while allele $A$ was predominant in European and Taoyuan black pigs (Table 2).

\begin{tabular}{|c|c|c|c|}
\hline Primer & Primer sequence & Size (bp) & Annealing temperature $\left({ }^{\circ} \mathrm{C}\right)$ \\
\hline SLC13A5-SNP1 & $\begin{array}{l}\text { F: CAGGCGTTGGGCTAAATG } \\
\text { R: TGATGAGTGGCAGCAGCAGTA }\end{array}$ & 997 & $60.0^{\circ}$ \\
\hline SLC13A5-SNP2 & $\begin{array}{l}\text { F: GCTTCTTCCTGCTGGTCG } \\
\text { R: CAGTGGCATCAGAGGTGTAA }\end{array}$ & 943 & $60.0^{\circ}$ \\
\hline SLC13A5-SNP3 & $\begin{array}{l}\text { F: TCTTTGAGGGTGTATCTTT } \\
\text { R: TCCTTCTATGGCACTATGT }\end{array}$ & 893 & $54.0^{\circ}$ \\
\hline SLC13A5-SNP4 & $\begin{array}{l}\text { F: GCCACCACTACCCTGTTC } \\
\text { R: TGCTTTCTTCTCAATCCC }\end{array}$ & 711 & $59.5^{\circ}$ \\
\hline SLC13A5-SNP5 & $\begin{array}{l}\text { F: CTCATTCCTGCGTCTTATTC } \\
\text { R: GCTGTGGGTGGTGTCATT }\end{array}$ & 815 & $60.0^{\circ}$ \\
\hline SLC13A5-qPCR & $\begin{array}{l}\text { F: TGACAGCAAAGACATCGTGAA } \\
\text { R: TTAGGGAAGGCAAATCCAAAC }\end{array}$ & 54 & $59.0^{\circ}$ \\
\hline GAPDH & $\begin{array}{l}\text { F: ATTTGGCTACAGCAACAGGGT } \\
\text { R: AAGTCAGGAGATGCTCGGTG }\end{array}$ & 172 & $59.0^{\circ}$ \\
\hline 5'-RACE & $\begin{array}{l}\text { GSP5: TGACGTAGGCACACCTGACAA } \\
\text { AUAP: GGCCACGCGTCGACTAGTAC }\end{array}$ & 557 & $55.0^{\circ}$ \\
\hline 3'-RACE & $\begin{array}{l}\text { GSP3: GGCGTCACGGCCTTTCTCTCCATGT } \\
\text { UPM : CTAATACGACTCACTATAGGGC }\end{array}$ & 1320 & $65.0^{\circ}$ \\
\hline
\end{tabular}

GSP = gene-specific primer; UPM and AUAP = universal and abridged universal amplification primers.
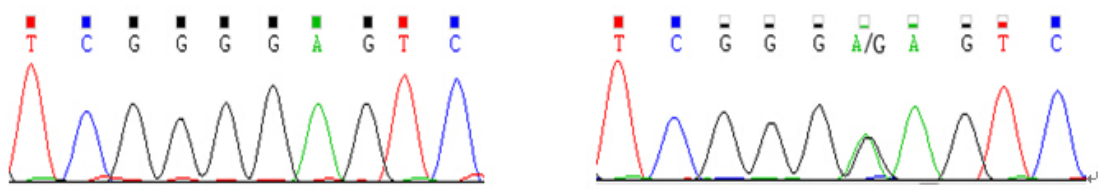

Figure 7. Sequencing maps of SNP for the SLC13A5 gene.

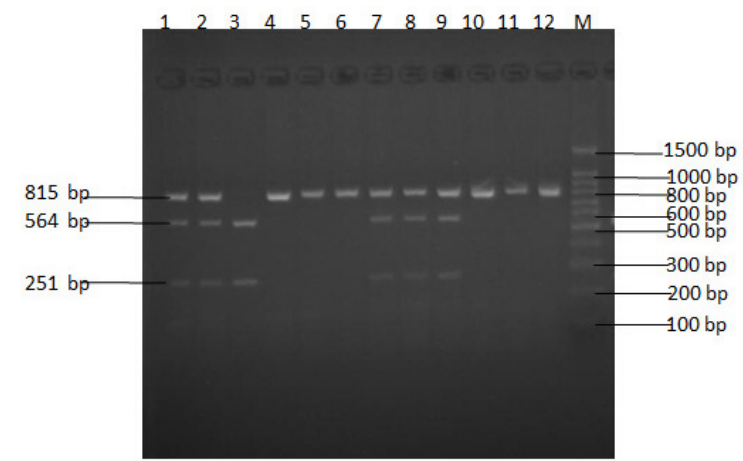

Figure 8. Electrophoresis patterns obtained from digestion with Bsu36I restriction enzyme. Lanes 1, 2, 7, 8, $9=A G$ genotype; lane 3 = GG genotype; lanes 4, 5, 6, 10, 11, 12 = AA genotype; lane $M=100$-bp DNA Ladder marker. 
Table 2. Genotypic distributions, allelic frequencies of the SLC13A5 gene of Bsu36I-PCR-RFLP genotype in five pig breeds.

\begin{tabular}{|c|c|c|c|c|c|c|}
\hline \multirow[t]{2}{*}{ Breed } & \multirow[t]{2}{*}{ Sample size } & \multicolumn{3}{|c|}{ Genotype frequency } & \multicolumn{2}{|c|}{ Allele frequency } \\
\hline & & $A A$ & $A G$ & $G G$ & $A$ & $G$ \\
\hline Yorkshire & 330 & 160 & 146 & 24 & 0.7061 & 0.2939 \\
\hline Daweizi & 80 & 17 & 34 & 29 & 0.4250 & 0.5750 \\
\hline Shaziling & 57 & 5 & 24 & 28 & 0.2982 & 0.7018 \\
\hline Ningxiang & 65 & 5 & 6 & 54 & 0.1231 & 0.8769 \\
\hline Taoyuan black & 112 & 71 & 37 & 4 & 0.7991 & 0.2009 \\
\hline
\end{tabular}

Allele frequency was accurate to fourth decimal point.

\section{Relationship between SNP and pig growth traits}

Least square means of data were calculated for the $A A, A G$, and $G G$ genotypes by general linear model. The genotype was not significantly correlated with age at $100 \mathrm{~kg}$ and corrected back fat thickness $(P>0.05$; Table 3$)$.

Table 3. Effect of three genotypes of the SLC13A5 gene on growth trait in pigs.

\begin{tabular}{lccc}
\hline Traits & \multicolumn{3}{c}{ Least square means \pm SD } \\
\cline { 2 - 4 } & $A A$ & $A G$ & $G G$ \\
\hline Age at $100 \mathrm{~kg}$ (day) & $170.0690 \pm 12.5784(136)$ & $170.3370 \pm 11.2826(120)$ & $172.2190 \pm 9.6174(21)$ \\
Correction backfat thickness (CF) $(\mathrm{mm})$ & $12.1378 \pm 3.1917(136)$ & $12.9885 \pm 3.3170(119)$ & $13.0911 \pm 3.929(22)$ \\
\hline
\end{tabular}

$C F$ is calculated by the formula $C F=1.0981$ ' $A /\{A+[B$ '(weight -100$)]\}$, for male pigs $A$ is 12.402 and $B$ is 0.106530 ; for female pigs $A$ is 13.706 and $B$ is 0.11964 .

\section{Tissue expression pattern of porcine SLC13A5}

The expression pattern of the SLC13A5 gene in ten different tissues of Shaziling pig and Yorkshire pig was analyzed by qPCR (Figure 9).

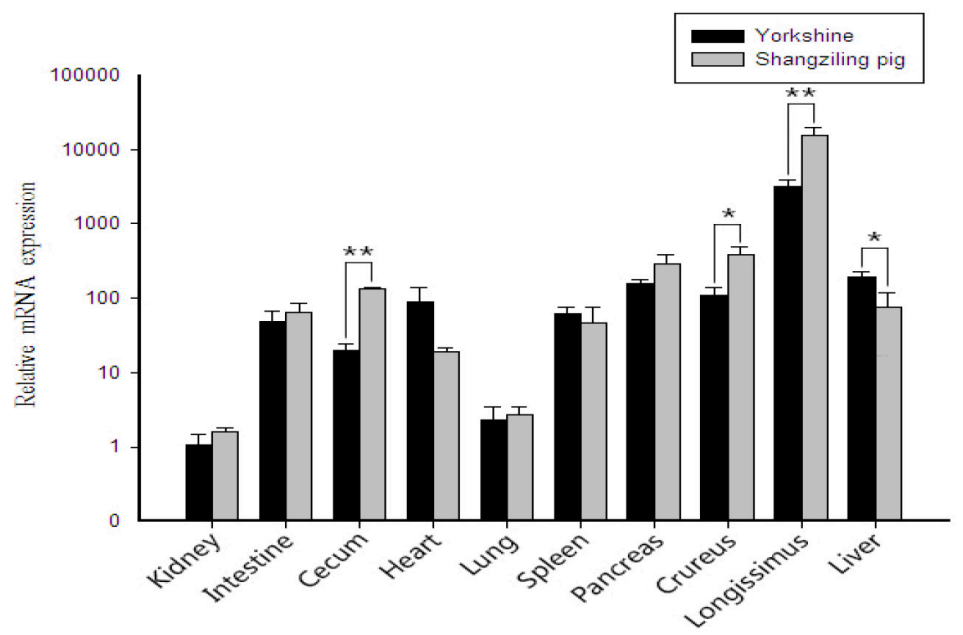

Figure 9. Expression profiles of the SLC13A5 gene in ten different tissues of Shaziling and Yorkshire breeds. Bars indicate the standard deviation of the mean. ${ }^{*} \mathrm{P}<0.05 .{ }^{* *} \mathrm{P}<0.01$. 
Expression levels of SLC13A5 in cecum and longissimus dorsi muscle were significantly different (both $\mathrm{P}<0.01$ ) in each of breeds, while significant in the liver and crureus muscle (both $\mathrm{P}<0.05$ ). In each of pig breeds, SLC13A5 expression levels in longissimus dorsi muscle differed significantly from those in the nine other tissues assessed $(P<0.05)$. Levels of SLC13A5 mRNA were high in the longissimus dorsi muscle, very low in kidney and lung, and moderate in other tissues.

\section{DISCUSSION}

In this study, porcine SLC13A5 coded 554 amino acids. The murine SLC13A5 comprised 572 amino acids, and its sequence was highly analogous to human and rat SLC13A5 (Inoue et al., 2002, 2004). Mouse SLC13A5 was approximately $23 \mathrm{~kb}$ in length, and contained twelve exons. Phylogenetic tree analysis indicated that the cattle, the sheep, and the pig formed one cluster, whereas the bonobo, the chimpanzee, the human, the white-cheeked gibbon, and the Rhesus constituted another. Finally, they formed a cluster with the wild pigeon and the ground tit. The porcine gene clustered with that of other mammals. SLC13A5 appears to be highly conserved during the process of species evolution.

The SLC13A5 protein was found to be a soluble protein through hydrophilic analysis (Pajor, 2006; Shulman and Helfand, 2011). In our study, bioinformatic analysis showed that 1-38 amino acids of porcine SLC13A5 existed in signal peptide, eight transmembrane regions were distributed among 45-67, 87-106, 121-140, 216-238, 253-275, 312-334, 354-373, and 488-510 amino acids; furthermore, there was a low-complexity region in 415-426 amino acids. We observed six serine (Ser), nine threonine (Thr), and three tyrosine (Tyr) sites, with a total of 18 potential phosphorylation sites.

In our study, porcine SLC13A5 mainly expressed in the longissimus dorsi muscle, and expressed in moderate levels in liver, crureus, and pancreas, low levels in kidney. Markovich and Murer (2004) reported that SLC13A5 expression was the highest in the gastrointestinal tract and epithelial cells of the kidney. It was expressed in normal human intestine (Weerachayaphorn and Pajor, 2008); however, mRNA of SLC13A5 was found not to be expressed in normal human intestine by RT-PCR (Arai et al., 2012). Gopal et al. (2015) reported that SLC13A5 also was predominantly expressed in the liver.

Our results showed that allele $G$ was predominant in Ningxiang and Shaziling breeds, but allele $A$ was predominant in European and Taoyuan black breeds. This SNP was not significantly correlated with age at $100 \mathrm{~kg}$ and corrected back fat thickness. The reason for this was the inadequate data. Also, SLC13A5 maybe was mainly expressed in the meat, not in fatty tissues. Further study is needed, however, to verify whether this SNP is associated with IMF formation.

\section{CONCLUSIONS}

In conclusion, we have characterized the SLC13A5 gene. This gene expressed in a high level in longissimus dorsi muscle of 25-day-old piglets. Our research provides an expression and structural basis for further study on functions of the SLC13A5 gene in pigs. Also, this study may provide the foundation for marker assistant selection (MAS) aimed at improving meat quality in pigs.

\section{Conflicts of interest}

The authors declare no conflict of interest. 


\section{ACKNOWLEDGMENTS}

Research supported by the National Science and High Technology Program of China (grant \#2011AA 1003 04), the Key Foundation of Education Department in Hunan (grant \#12A060), and the Science and Technology Project of Hunan Province (Grand \#2014NK4135).

\section{REFERENCES}

Arai E, Chiku S, Mori T, Gotoh M, et al. (2012). Single-CpG-resolution methylome analysis identifies clinicopathologically aggressive CpG island methylator phenotype clear cell renal cell carcinomas. Carcinogenesis 33: 1487-1493.

Bergeron MJ, Clémençon B, Hediger MA and Markovich D (2013). SLC13 family of $\mathrm{Na}^{+}$-coupled di- and tri-carboxylate/sulphate transporters. Mol. Aspects Med. 34: 299-312.

Biermann AD, Yin T, König von Borstel UU, Rübesam K, et al. (2015). From phenotyping towards breeding strategies: using in vivo indicator traits and genetic markers to improve meat quality in an endangered pig breed. Animal 9: 919-927.

Birkenfeld AL, Lee HY, Guebre-Egziabher F, Alves TC, et al. (2011). Deletion of the mammalian INDY homolog mimics aspects of dietary restriction and protects against adiposity and insulin resistance in mice. Cell Metab. 14: $184-195$.

Chen X, Feng Y, Yang WJ, Shu G, et al. (2013). Effects of dietary thiazolidinedione supplementation on growth performance, intramuscular fat and related genes mRNA abundance in the longissimus dorsi muscle of finishing pigs. Asian Australas. J. Anim. Sci. 26: 1012-1020.

Ding XL, Ding YY, Zhang CH, Huang L, et al. (2011). Polymorphisms and sequencing analysis in 5'upstream and intron 2 region of $H$-FABP gene in Anhui Wannan black pig population. J. Anim. Vet. Adv. 10: 1568-1572.

Etcheverry A, Aubry M, de Tayrac M, Vauleon E, et al. (2010). DNA methylation in glioblastoma: impact on gene expression and clinical outcome. BMC Genomics 11: 701.

Gopal E, Babu E, Ramachandran S, Bhutia YD, et al. (2015). Species-specific influence of lithium on the activity of SLC13A5 $(\mathrm{NaCT})$ : lithium-induced activation is specific for the transporter in primates. J. Pharmacol. Exp. Ther. 353: 17-26.

Inoue K, Zhuang L and Ganapathy V (2002). Human $\mathrm{Na}^{+}$-coupled citrate transporter: primary structure, genomic organization, and transport function. Biochem. Biophys. Res. Commun. 299: 465-471.

Inoue K, Fei YJ, Zhuang L, Gopal E, et al. (2004). Functional features and genomic organization of mouse NaCT, a sodiumcoupled transporter for tricarboxylic acid cycle intermediates. Biochem. J. 378: 949-957.

Li L, Li H, Garzel B, Yang H, et al. (2015). SLC13A5 is a novel transcriptional target of the pregnane X receptor and sensitizes drug-induced steatosis in human liver. Mol. Pharmacol. 87: 674-682.

Luo W, Cheng D, Chen S, Wang L, et al. (2012). Genome-wide association analysis of meat quality traits in a porcine large white $x$ Minzhu intercross population. Int. J. Biol. Sci. 8: 580-595.

Ma J, Ren J, Guo Y, Duan, Y, et al. (2009) .Genome-wide identification of quantitative trait loci for carcass composition and meat quality in a large-scale White Duroc x Chinese Erhualian resource population. Anim Genet. 40: 637-647.

Ma J, Yang J, Zhou L, Zhang Z, et al. (2013). Genome-wide association study of meat quality traits in a White Duroc $x$ Erhualian $\mathrm{F}_{2}$ intercross and Chinese Sutai pigs. PloS one 8: e64047.

Madeira MS, Costa P, Alfaia CM, Lopes PA, et al. (2013). The increased intramuscular fat promoted by dietary lysine restriction in lean but not in fatty pig genotypes improves pork sensory attributes. J. Anim. Sci. 91: 3177-3187.

Markovich D and Murer H (2004). The SLC13 gene family of sodium sulphate/carboxylate cotransporters. Pflugers Arch. 447: 594-602.

Martinez-Beamonte R, Navarro MA, Guillen N, Acin S, et al. (2011). Postprandial transcriptome associated with virgin olive oil intake in rat liver. Front. Biosci. 3: 11-21.

Pajor AM (2006). Molecular properties of the SLC13 family of dicarboxylate and sulfate transporters. Pflugers Arch. 451: $597-605$.

Ren ZQ, Wu WJ, Liu WH, Zheng R, et al. (2014). Differential expression and effect of the porcine ANGPTL4 gene on intramuscular fat. Genet. Mol. Res. 13: 2949-2958.

Ros-Freixedes R, Reixach J, Bosch L, Tor M, et al. (2014). Genetic correlations of intramuscular fat content and fatty acid composition among muscles and with subcutaneous fat in Duroc pigs. J. Anim. Sci. 92: 5417-5425.

Sato S, Ohnishi C, Kikuchi T, Kohira K, et al. (2014). Evaluation of quantitative trait loci affecting intramuscular fat and reproductive traits in pigs using marker-assisted introgression. Anim. Genet. 45: 799-807.

Schwab CR, Baas TJ, Stalder KJ and Mabry JW (2006). Effect of long-term selection for increased leanness on meat and eating quality traits in Duroc swine. J. Anim. Sci. 84: 1577-1583.

Schworer R and Lorenz D (1995). Selection of intramuscular fat in Swiss pig breeds and the importance of fatty tissue quality. Proceeding of 2nd Dummerstorf Muscle Workshop on Growth and Meat Quality, Rostock. 
Shulman Gl and Helfand SL (2011). Indy knockdown in mice mimics elements of dietary restriction. Aging 3: 701.

Tamura K, Peterson D, Peterson N, Stecher G, et al. (2011). MEGA5: Molecular Evolutionary Genetics Analysis using Maximum Likelihood, Evolutionary Distance, and Maximum Parsimony Methods. Mol. Biol. Evol. 28: 2731-2739.

Weerachayaphorn $J$ and Pajor AM (2008). Identification of transport pathways for citric acid cycle intermediates in the human colon carcinoma cell line, Caco-2. Biochim. Biophys. Acta 1778: 1051-1059.

Wood JD, Richardson RI, Nute GR, Fisher EV, et al. (2004). Effects of fatty acids on meat quality: A review. Meat Sci. 66: 21-32.

Wood JD, Enser M, Fisher AV, Nute GR, et al. (2008). Fat deposition, fatty acid composition and meat quality: a review. Meat Sci. 78: 343-358.

Xiong Q, Chai J, Deng C, Jiang S, et al. (2012). Characterization of porcine SKIP gene in skeletal muscle development: polymorphisms, association analysis, expression and regulation of cell growth in C2C12 cells. Meat Sci. 92: 490-497. 